\title{
Study of G.P. Zones in a Binary Al-10 at \% Zn Alloy
}

\author{
By Masahiko Morinaga*, Kuo-Shung Liu**, Yōichiro Tomita*** \\ and Yotaro Murakami****
}

\begin{abstract}
X-ray diffuse scatterings around the direct beam (i.e. X-ray small angle scattering) and around the Bragg angles of (111) and (200), were measured with powder samples of an $\mathrm{Al}-10$ at $\% \mathrm{Zn}$ alloy containing G.P. zones.

The diffuse scatterings around the Bragg angles were caused by G.P. zones. Their scattering intensities were strongly dependent on zone-size and their scattering profiles changed from symmetrical to asymmetrical around the Bragg angle with increasing zone-size from $R_{G}=31 \AA$ to $52 \AA$ ( $R_{G}$ : Guinier radius). The asymmetrical diffuse scatterings were interpretable as being due to the size effect scatterings which reflect the static displacement of atoms from the lattice points (i.e. static distortion). The size effect scatterings became more intensive with the zone size.
\end{abstract}

(Received June 19, 1973)

\section{Introduction}

There have been many investigations on G.P. zones in several age-hardening aluminum alloys, especially in the $\mathrm{Al}-\mathrm{Zn}$ and $\mathrm{Al}-\mathrm{Ag}$ alloys, where G.P. zones have been considered to be almost spherical because of the similarity of atomic radius between solute and solvent atoms. Gragg and Cohen ${ }^{(1)}$ proposed recently that below $170^{\circ} \mathrm{C}$ G.P. zones in an $\mathrm{Al}-\mathrm{Ag}$ alloy are octahedral in shape and attributed its cause to the distortion due to the slightly different atomic radius between aluminum and silver atoms. The above considerations concerning G.P. zone itself and the static distortion accompanied with a zone may also be necessary in an Al-Zn alloy. Because, the static distortion is expected to be larger than that in an $\mathrm{Al}-\mathrm{Ag}$ alloy since the difference of atomic radius between aluminum and zinc atoms is larger than that of an $\mathrm{Al}-\mathrm{Ag}$ alloy. In fact, it has been reported that the G.P. zone in an $\mathrm{Al}-\mathrm{Zn}$ alloy changes its shape from spherical to ellipsoidal at the critical zone radii of $30 \sim 35 \AA^{(2)}$. This shape change results from the anisotropy of a strain field of G.P. zones. The ellipsoidal G.P. zones have a preferred $\{111\}$ orientation and show a rhombohedral distorted structure with a rhombohedral strain field ${ }^{(3)(4)}$.

In the present experiment, considering the above results, the nature of G.P. zones in an $\mathrm{Al}-10$ at $\% \mathrm{Zn}$ alloy was examined in detail through measurements of $\mathrm{X}$-ray diffuse scatterings at both small and high angles by the diffractometer method. The difference between these two diffuse scatterings is illustrated in Fig. $1^{(5)}$, in which (a) is the profile of diffuse scattering due to only clustering, i.e. due to the local distribution of

* Graduate School, Kyoto University, Kyoto, Japan. Present address: Department of Materials Science, The Technological Institute, Northwestern University, Evanston, Illinois 60201, U.S.A.

** Faculty of Engineering, Kyoto University, Kyoto, Japan. Present address: National Tsing Hua University, Taiwan, China.

*** Graduate School, Kyoto University, Kyoto Japan.

**** Department of Metallurgy, Faculty of Engineering, Kyoto University, Kyoto, Japan.

Trans. JIM

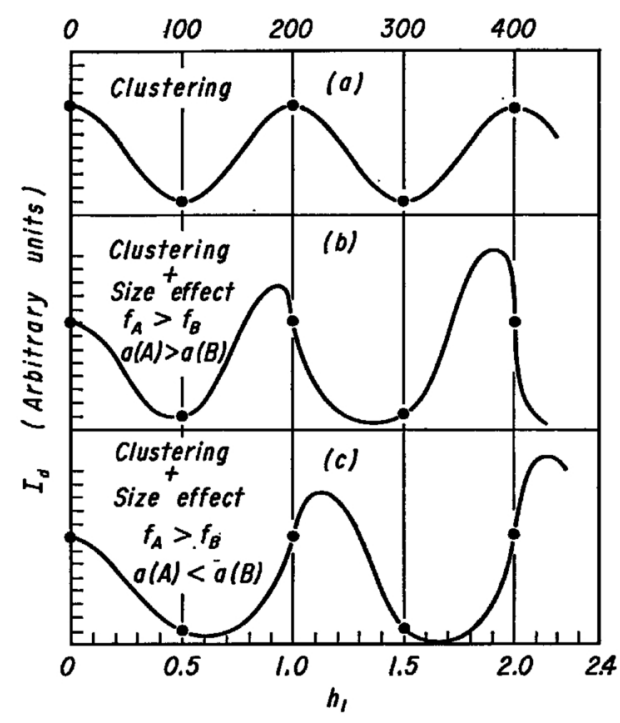

Fig. 1 Idealized diffuse scattering along $\langle 100\rangle$ for a binary single crystal with clustering and size effect.

solute and solvent atoms with respective atomic scattering factors $f_{A}$ and $f_{B}$. In addition to this scattering, the size effect scattering due to the displacements of atoms from the lattice points because of the difference of lattice spacings $a(A)$ and $a(B)$ (i.e. static distortion), distorts the scattering profile as shown in (b) and (c). As indicated in the figure, the peak position of scattering profile shifts according to the sign of inequality between $f_{A}$ and $f_{B}$ or $a(A)$ and $a(B)$. In an Al-Zn alloy used, inequalities $f_{\mathrm{Zn}}>f_{\mathrm{Al}}$ and $a_{\mathrm{Zn}}<a_{\mathrm{Al}}$ (as the lattice spacing of solid solution contracts with increasing zinc concentration) are satisfied, and consequently case (c) is expected. It should be noticed that the small angle scattering is scarcely influenced by the size effect, but the diffuse scatterings around the Bragg angles are strongly influenced by this effect.

Thus, through the measurements of diffuse scattering at small and high angles, the nature of G.P. zones can be made clear. In this experiment, it was confirmed that the diffuse scattering around the Bragg angle was strongly dependent on zone size, and that the size effect scattering increased with the zone size.

1974 Vol. 15 


\section{Experimental Procedure}

The material used was an aluminum alloy containing 10 at $\% \mathrm{Zn}$ which was prepared from aluminum and zinc, each having a purity better than $99.99 \%$. From this material, the powder samples, the grain size of which was controlled to be less than $53 \mu$ by passing through a sieve, were prepared for the measurements of X-ray diffuse scatterings at small and high angles. The powder samples were filled in a silica tube of about $1.5 \mathrm{~mm}$ I.D. and vacuum sealed. Each sample was annealed at $500^{\circ} \mathrm{C}$ for $30 \mathrm{~min}$ for the release of internal stress. Merz et al. ${ }^{(6)}$ reported that the zone size becomes constant at a certain value which depends on the quenching method and the quenching temperature. In order to obtain various zone sizes air quenching and quenching into iced water were done and the quenching temperatures were also varied, i.e. 300,400 and $500^{\circ} \mathrm{C}$. The agings were performed in an iced water bath $\left(\sim 0^{\circ} \mathrm{C}\right)$ for $38 \mathrm{hr}$, which is long enough for the zones to become constant in size. Therefore, it may be thought that the aging during the $\mathrm{X}$-ray measurement at room temperature can be neglected.

The X-ray used was Ni-filtered $\mathrm{Cu} K_{\alpha}$ radiation and the scattering intensities at small and high angles were counted by the fixed time method. The X-ray intensities for the powder samples are influenced by the packings of powders. To eliminate this undesired effect all scattering intensities were normalized by the intensity of the (111) Bragg reflection. The intensity of the Bragg reflection is likely to change according to the state of precipitates, but this change may be neglected at small diffraction angle such as the (111) reflection $^{(7)}$. The counted X-ray spectrum was subtracted by that for the sample in which the G.P. zones were dissolved (reverted) by aging at $215^{\circ} \mathrm{C}$ for $1 \mathrm{~min}$. In the spectrum of the diffuse scattering obtained by this correction, the errors caused by the X-ray apparatus and the diffuse scatterings due to the thermal displacement of atoms from lattice points are roughly eliminated.

The scattering measurements at very small angles were done by the transmission technique using an apparatus with a Kratky slit ${ }^{(8)}$.

\section{Results and Discussion}

The typical X-ray diffraction pattern near the (111) and (200) reflections is shown in Fig. 2. This measured $\mathrm{X}$-ray intensity curve is asymmetrical around each Bragg angle of (111) and (200). The real diffuse scattering intensities were obtained from these intensities by subtracting the intensities of reverted sample at each angle. In Fig. 3, typical X-ray diffuse scattering spectra are shown for the specimen aged at $0^{\circ} \mathrm{C}$ for $38 \mathrm{hr}$ after water-quenching from $300^{\circ} \mathrm{C}$. Thus, the diffuse scatterings were observed around the direct beam and every Bragg angle. By applying the Guinier approximation to the $\mathrm{X}$-ray small angle scattering

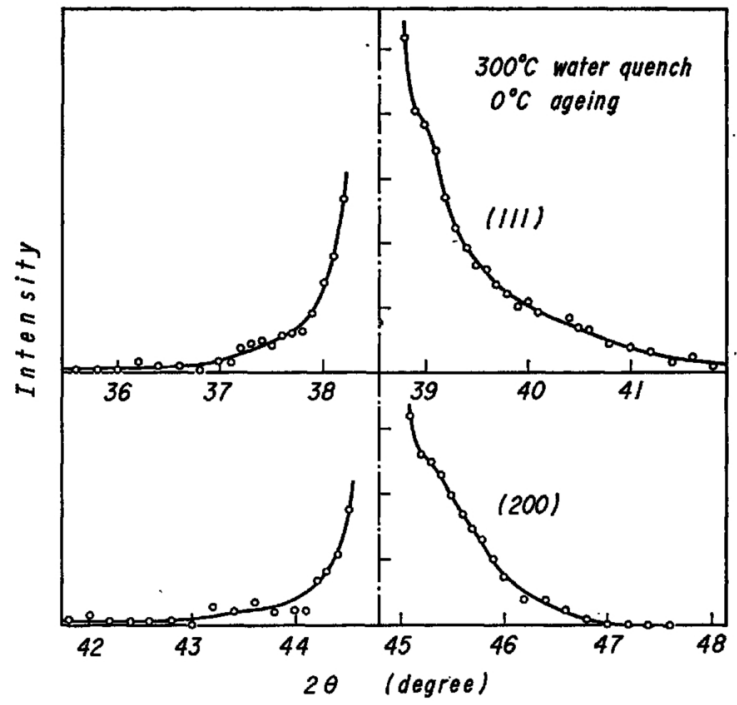

Fig. 2 Typical X-ray diffraction pattern near the Bragg angles of (111) and (200).

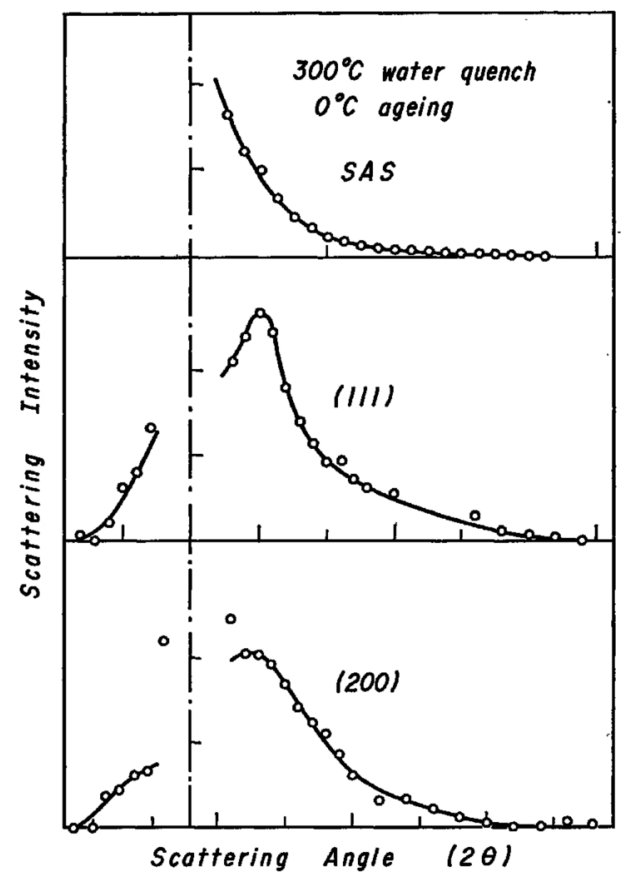

Fig. 3 The diffuse scattering curves around the direct beam (SAS) and Bragg angles of (111) and (200).

(SAS) curve the zone radius was determined to be about $52 \AA$. The same analyses were done for the various specimens which were quenched into iced water or air-quenched from various temperatures. These results are shown in Figs. 4 and 5 for the diffuse scatterings around (111) and (200) respectively. In each figure, the zone radius determined from the Guinier approximation is given. From these figures, two experimental results should be noticed. The first one is that the diffuse scattering observed around the Bragg angle depends largely on the zone size. The same result was also confirmed for the specimens aged at $38^{\circ} \mathrm{C}$ for 20 $\mathrm{hr}$. The zone size dependence of the diffuse scattering at high angles has not found in previous investigations. The second one is that the peak position of diffuse scattering curve shifts to the Bragg angle with increas- 


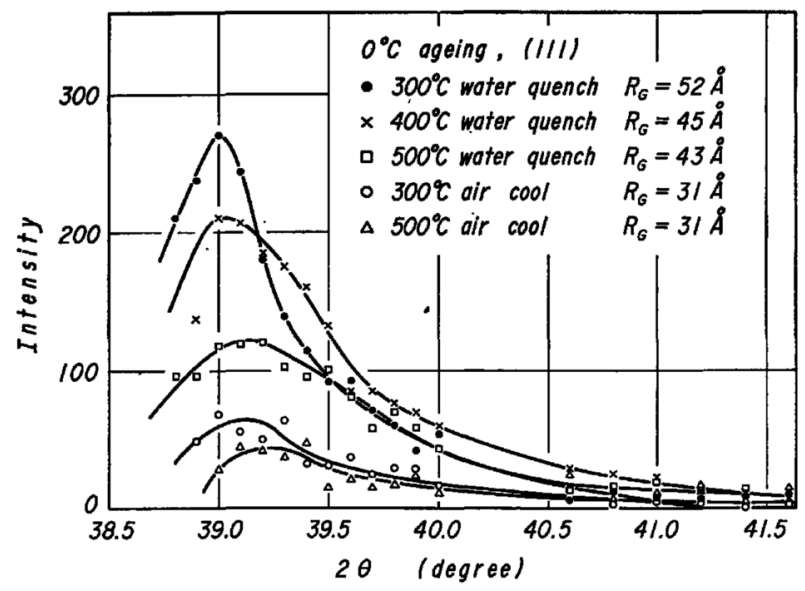

Fig. 4 The diffuse scattering curves at high angle side of (111) Bragg angle for various specimens aged at $0^{\circ} \mathrm{C}$ for $38 \mathrm{hr}$.

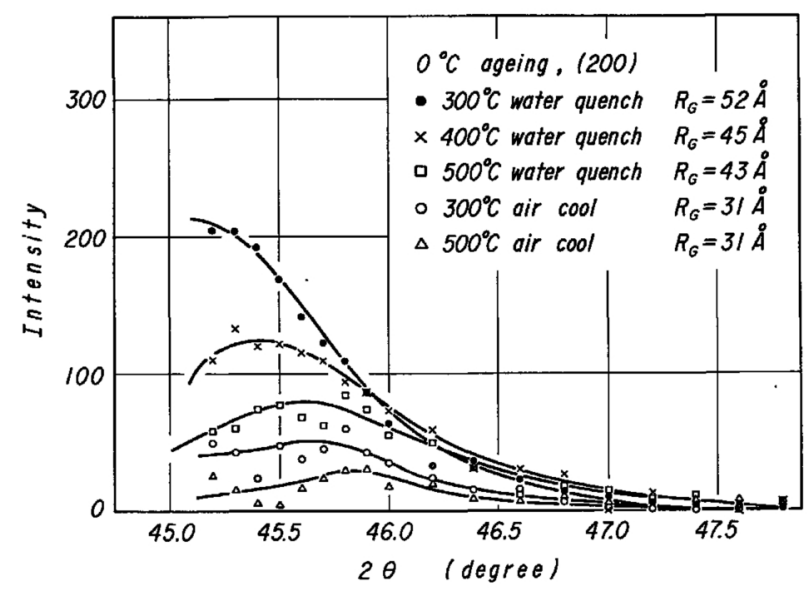

Fig. 5 The diffuse scattering curves at high angle side of (200) Bragg angle for various specimens aged at $0^{\circ} \mathrm{C}$ for $38 \mathrm{hr}$.

ing zone size. In the X-ray small angle scattering curve, the occurrence of the same shift is well known, which has been explained on the analogy of Scherrer's equation for small particle that the width of diffuse halo is reversely proportional to the particle size. The diffuse scattering around the Bragg angle is the convolution of the scatterings due to the clustering and due to the size effect. So, it may be impossible to determine which scattering is the origin of this peak shift, but the increase of the clustered region, i.e. the zone growth may be dominant. To examine the scattering due to the size effect the diffuse scattering profiles at both sides of the (111) Bragg angle are shown in Fig. 6. As seen in this figure, at the zone size $R_{G}=31 \AA$, the almost same diffuse scatterings were observed at both sides of the Bragg angle. This means that the size effect scattering is small. It should be noticed that in this $\mathrm{Al}-\mathrm{Zn}$ alloy the diffuse scatterings at both sides of the Bragg angles were caused by G.P. zones with a distinct interface with the matrix, not by the modulated structure which has usually been connected with the side-bands of an X-ray diffraction pattern. The G.P. zones have the solute concentration $\left(m_{1}\right)$ and are suspended in the medium of concentration $m_{2}$. The values of $m_{1}$ and $m_{2}$ are represented by the metastable

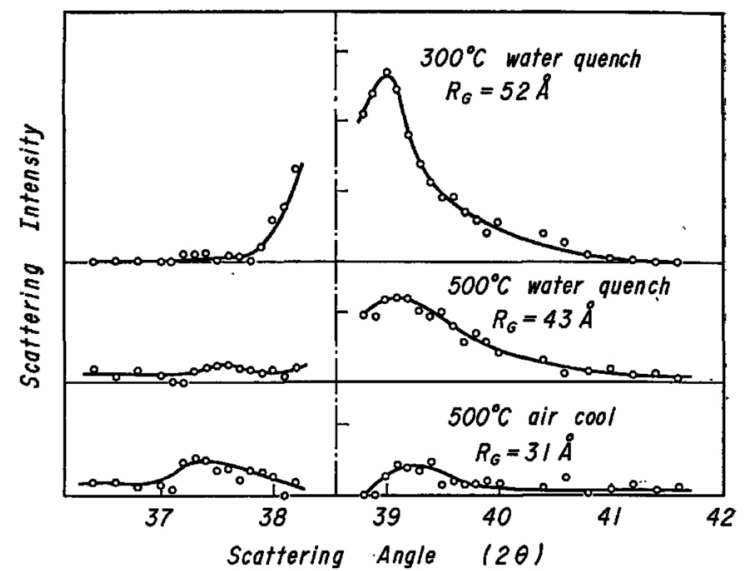

Fig. 6 The diffuse scattering curves around (111) Bragg angle for the specimens with various zone radii.

miscibility gap: The values at $0{ }^{\circ} \mathrm{C}$ are $m_{1}=0.75$, $m_{2}=0.012$. The volume fraction of G.P. zones is about 0.12 . As the zone size increases, the diffuse scattering profile becomes asymmetrical. This can be interpreted as being due to the size effect scattering. The degree of asymmetry of the profile increases with the zone size, which means that the scattering due to the static displacements of atoms from the lattice points (i.e. static distortion) increases with the zone size. Previous investigation ${ }^{(2)}$ showed that the zone changes its shape from spherical to ellipsoidal at the critical zone radii of $30 \sim 35 \AA$, due to the anisotropy of strain field. This critical radius coincides with the radius at which the scattering profile becomes asymmetrical. However, it is not clear whether the increase of the size effect scattering with the zone size is due to the increase of the degree of distortion or due to the extention of the distorted regions.

\section{Conclusions}

To study the G.P. zones in a binary $\mathrm{Al}-10$ at $\% \mathrm{Zn}$ alloy, the X-ray small angle scattering and the diffuse scattering around the Bragg angles of (111) and (200) were measured with the powder samples.

The diffuse scatterings around the Bragg angles were caused by the G.P. zones. Their scattering intensities depended mainly on the zone size. The change of this scattering profile with the zone size was remarkable. At the zone radius of about $31 \AA$, the profile was symmetrical around the Bragg angle, but as the zone radius increases the profile became asymmetrical. This means that the size effect scattering due to the static distortion inside and around the zone became more intensive with the zone size. The zone radius at which the profile changed from symmetrical to asymmetrical coincided with the critical radius where the zone changes in shape from spherical to ellipsoidal.

\section{Acknowledgments}

The authors should like to thank Prof. Paul H. Shingu for his continued interest and reading the manuscript. Also, they would like to thank Prof. 
J. B. Cohen and Prof. R. W. Gould for reading the manuscript.

\section{REFERENCES}

(1) J. E. Gragg, Jr. and J. B. Cohen: Acta Met., 19 (1971), 507.

(2) W. Merz and V. Gerold: Z. Metallk., 57 (1966), 607.

(3) W. Merz, T. Anantharaman and V.Gelord: Phys. Status Solidi, 8 (1965), K5.
(4) H. Auer, V. Gerold, H. Haberkorn and W. Zurn: Phys. Status Solidi, 6 (1964), K91.

(5) S. C. Moss and B. L. Averbach: Small-Angle X-ray Scattering, Gordon and Breach, New York, (1968), 335.

(6) W. Merz, V. Gerold and O. Kawano: Z. Metallk., 61 (1970), 103.

(7) I. Nitta: X-ray Crystallography (Japanese), Maruzen, Japan, (1961), 517.

(8) O. Kratky: Z. Electrochemie., 58 (1954), 49. 